\title{
Pharmaceutical care in paediatric intensive care unit: activities and interdisciplinary learning in a Spanish hospital
}

\author{
Lara Echarri-Martínez, ${ }^{1}$ C M Fernández-Llamazares, ${ }^{1}$ Silvia Manrique-Rodríguez, ${ }^{1}$ Isabel García-López, ${ }^{1}$ \\ J López-Herce, ${ }^{2}$ María Sanjurjo-Sáez ${ }^{1}$
}

Objectives To describe and quantify the clinical and logistic activities of a resident pharmacist in a paediatric intensive care unit (PICU) and to identify the key areas for improvements in training.

Methods A prospective quasi-experimental cross-sectional study was conducted in an 11-bed PICU over a 3-month period. Pharmacist recommendations (clinical interventions) were made on patient care during the validation stage of medical orders. All interventions performed were recorded in a database and included the following information: reason for the intervention, clinical significance and acceptance by the physician. A record was also kept of the number of interventions per patient-day and the number of drugs whose profiles for dispensing from an automatic cabinet automatic dispensing system (ADS) were modified.

Results There were 40 interventions (13\% recommendations issued in the absence of error, 59\% concerned drug safety issues, $20 \%$ related to efficacy and $8 \%$ to the indication), of which $72.5 \%$ were classified as significant or very significant; $95 \%$ were accepted by the physician. There were 4.0 interventions per 100 patient-days. No single drug was involved in more than $5 \%$ of interventions. The profiles of $16.6 \%$ of the drugs included in the ADS were modified.

Conclusions The action of the pharmacist enabled prevention of prescription errors of critically ill paediatric patients. It is necessary to broaden and systematise clinical training in this discipline in order to identify a larger number of negative outcomes associated with medications.

\section{Introduction}

In recent years pharmaceutical practice has become orientated towards achieving therapeutic results and a rational use of drugs. In order to further develop these activities in the complexity of the hospital setting, pharmacists need to become directly involved in patient care through incorporation into the care team in different hospital units. In 1999 the National Specialties Commission in Spain added a fourth year to the hospital pharmacists' training programme established by Royal Decree $2708 / 82$ on 15 October. ${ }^{1}$ The objective of this fourth year of residency was 'to perform the specific duties of the pharmacist in contact with patients and



${ }^{1}$ Hospital Pharmacy, Gregorio Marañón University General Hospital, Madrid, Spain

2Pediatric Intensive Care Unit, Gregorio Marañón University General Hospital, Madrid, Spain

Correspondence to Lara Echarri-Martínez, Hospital Pharmacy, Hospital General Universitario Gregorio Marañón, Servicio de Farmacia, c/Dr Esquerdo 46, Madrid 28007, Spain; larott@hotmail.com to promote integration with the rest of the care team'. The resident thus had to take on the responsibility of ensuring that treatments administered to patients were both appropriate and effective. ${ }^{2}$ In order to achieve these objectives, the Spanish Society of Hospital Pharmacy drew up strategic training directives which described the need to equip fourth-year residents with a systematic methodology and specific measurement variables to be able to structure their work and thus contribute positively to a patient's pharmacotherapeutic strategy in each type of hospital unit such as oncology, the surgical specialties, infectious diseases units and paediatrics. ${ }^{3}$

As with the adult population, the later development of clinical pharmacy as a specialty in Spain compared with other countries has meant that few studies have focused on the integral role of the pharmacist in paediatrics, and there is no specific training schedule for residents that defines the abilities to be acquired. In addition, the continuous developmental changes in the paediatric population and the immaturity of their physiological systems lead to different care needs from those of adults and a distinct therapeutic approach with regard to drugs, pharmaceutical forms, excipients and administration techniques. These peculiarities make children more vulnerable than adults to medication errors, as has been demonstrated in a number of studies. ${ }^{45}$ Furthermore, patients admitted to paediatric intensive care units (PICUs) are characterised by certain pharmacokinetic peculiarities due to disturbances in one or more of their vital organs. ${ }^{6}$ Intensive care units are particularly susceptible to medication errors. ${ }^{7}$ This fact makes them ideal areas for implementing new strategies related to clinical activities (pharmacotherapeutic follow-up) and also to optimise distribution systems in order to improve safety in hospitals and reduce medication errors. ${ }^{8}$

The aim of this study is to describe and quantify the clinical and logistic activities of a resident pharmacist in a PICU. We also identify the key areas for improvements in training.

\section{Methods}

This prospective cross-sectional study was performed in the 11-bed PICU of our hospital in Madrid, Spain over a 3-month rotation in the unit (from 1 October to 31 December 2009). Logistic activities and clinical interventions made by a resident pharmacist during a paediatric pharmacist's rotation in the PICU were included. A clinical specialist pharmacist in the Central Pharmacy provided support to the resident but only activities and recommendations 
made by the resident were included. Attendance in the unit was from Monday to Friday, 08:00 to 15:00 h. Activities performed outside this time period were excluded because after 15:00 $\mathrm{h}$ the resident works in the Central Pharmacy.

The requirement for informed consent was waived because the participation of the pharmacist integrated into the unit was considered by all the team in the department as part of the routine work of the PICU, and the rest of the health workforce is required to preserve the confidentiality and use data only for purpose of scientific publication.

\section{Clinical activities}

After the first morning session and full ward round in the intensive care unit, the medical staff prescribed patient treatment electronically using the Visual Limes Prescriplant programme. The pharmacist then validated the physicians' orders. Validation focused on confirmation of the correct dose of the drug according to the weight and height of the child, the suitability of the drug prescribed and its indication, the dose adjustments recommended in the literature according to the characteristics of the patient, the absence of contraindications and clinically significant interactions, and the proposal of alternative drugs for those not included in the hospital's pharmacotherapeutic guidelines, recommending available equivalents. If clarification was required, the pharmacist wrote a note appended to the electronic prescription or contacted the responsible physician by telephone.

All interventions were recorded in a Microsoft Access database designed in the Pharmacy Department to quantify and evaluate the activity of the pharmacists in each clinical unit; the database had been validated by pharmacists with various levels of specialization, observing an acceptable degree of concordance. ${ }^{9}$ Clinical interventions were defined as those recommendations issued when a prescription error was detected and interventions in which the additional information provided on the use of a drug may have avoided a medication error. The resolution of consultations made by the health professionals of the unit about drug dosage and administration and clarification of incomplete physicians' orders were not considered as interventions unless they could have led to an error in the nursing staff's interpretation at the time of drug administration. Other prescription errors also excluded from the study were those involving parenteral or enteral nutritional, intravenous chemotherapy preparations and dose modifications made by the pharmacokinetics unit. Quantification and descriptive statistical analysis of the data gathered in the MS Access database was performed using MS Excel.

The design concept of the database was based on the methodology of two high-impact studies on the development of recording systems for pharmacists' interventions. ${ }^{1011}$ The following measurement variables were included: negative outcomes associated with medication (NOM) detected and reason for the intervention and clinical significance, acceptance of intervention and drug involved in the intervention.

\section{Negative outcomes associated with medication (NOM) detected}

The NOMs were classified into six categories (box 1). In addition, NOMs were defined as avoided (if the medication error could have caused harm but the drug was not administered to the patient) or real (if the drug involved in the medication error was administered to the patient and led to the corresponding repercussions).

Reason for the intervention and clinical significance

The recommendation made by the pharmacist to the responsible physician was recorded. The different motives for intervention are listed in table 1, together with the clinical significance of each of the motives based on a slightly modified Overhage scale. ${ }^{11}$

\section{Acceptance of the intervention}

The prescribing physician's acceptance of the recommendation issued was evaluated over the following $24 \mathrm{~h}$ and was classified as accepted, not accepted or not evaluable (when it was not possible to assess acceptance due to transfer of the patient to another hospital ward); these events were excluded from the analysis.

\section{Drug involved in the intervention}

Both the trade name and the active substance were recorded. Drugs were included whether or not they appeared in the hospital's pharmacotherapeutic guidelines.
The number of interventions per patientday was used as the indicator of activity, which was calculated using data from Farhos Unidosis $^{\mathrm{TM}}$ (a database used in the pharmacy Department). This indicator shows the number of physician orders validated and the number of patients admitted during the study period (patient-days were defined as active prescriptions per occupied bed).

\section{Logistic activities}

The main medication store, which was already implemented before this study in the PICU, was the Pyxis automatic dispensing system (ADS) which was linked via an interface to the prescription profile of the patient. In this way, the dispensing system received the information of the medical prescription in real time. This function of the system linked to the patient profile provided an additional control, allowing access only to medications prescribed and validated by the pharmacy department. However, drugs were categorised into different profiles (groups 1 and 2) according to the therapeutic group to which they belonged and their use in the unit. Drugs included in group 1 required an electronic prescription and validation by the pharmacist in order for nurses to be able to access the drug. Those in group 2, for urgent treatment or for nursing care (PRN, as needed) could be accessed by the nurse without prior prescription by the physician or validation by the pharmacist.

The resident updated and reviewed drugs included in the dispensing system based on a drug list updated in July 2009 and supplied by the dispensing and logistics service of the pharmacy department. In an initial phase, the drug profiles were reviewed with the head of the nursing staff in the unit and another experienced nurse in order to identify discrepancies between the established profile of each of the drugs in the ADS and its actual use in the PICU. In a second
Box 1 Classification of negative outcomes associated with medication (NOMs) according to the Third Consensus of Granada (2007)

Necessity

Untreated health problem: the patient has a health problem as a consequence of not receiving the medicine that he needs

Effect of unnecessary medicine: the patient has a health problem as a consequence of receiving a medicine that he does not need

Effectiveness

Non-quantitative ineffectiveness: the patient has a health problem associated with the non-quantitative ineffectiveness of the medication

Quantitative ineffectiveness: the patient has a health problem associated with the quantitative ineffectiveness of the medication

Safety

Non-quantitative safety problem: the patient has a health problem associated with a non-quantitative safety problem with the medication

Quantitative safety problem: the patient has a health problem associated with a quantitative safety problem with the medication 
Table 1 Clinical significance according to the motive for the intervention

\begin{tabular}{|c|c|c|c|}
\hline Type of intervention & Clinical significance & n & $\%$ \\
\hline Economic saving & No error & 0 & 0.0 \\
\hline Documented drug allergy & Serious & 1 & 2.5 \\
\hline Clarification of the physician's order/request for information & No error & 5 & 12.5 \\
\hline Therapeutic duplication & Significant & 1 & 2.5 \\
\hline Dose error: resulting in potentially toxic concentrations & Serious & 3 & $17.5^{*}$ \\
\hline Dose error: 1.5-10 times higher than normal (if drug had a normal therapeutic range) & Significant & 1 & \\
\hline Dose error: $1.5-4$ times higher than normal (if drug had a narrow therapeutic range) & Significant & 0 & \\
\hline Dose error: 10 times higher than normal (if drug had a narrow therapeutic range) & Potentially lethal & 1 & \\
\hline Dose error: 10 times higher than normal (if drug had a normal therapeutic range) & Serious & 1 & \\
\hline Dose error: 4-10 times higher than normal (if drug had a narrow therapeutic range) & Serious & 1 & \\
\hline Dose error: very low in non-potentially life-saving drugs & Significant & 3 & 7.5 \\
\hline Dose error: very low in potentially life-saving drugs & Potentially lethal & 0 & \\
\hline Repackaging error/physician's order led to error of dispensing & Serious & 0 & 0.0 \\
\hline Pharmaceutical form inappropriate or not available & Minor & 2 & 5.0 \\
\hline Illegible, ambiguous or incomprehensible abbreviations & Minor & 0 & 0.0 \\
\hline Non-adherence to hospital policy & Minor & 0 & 0.0 \\
\hline Incomplete information in the physician's order & Minor & 1 & 2.5 \\
\hline Interaction: clinically significant, requiring follow-up & Significant & 2 & 5.0 \\
\hline Interaction: combination contraindicated & Potentially lethal & 0 & \\
\hline Inappropriate dosage interval & Significant & 6 & 15.0 \\
\hline Drug not supplied by company & No error & 0 & 0.0 \\
\hline Drug not indicated (indication not studied or with no evidence of use) & Serious & 0 & 0.0 \\
\hline Drug not included in guidelines & Minor & 0 & 0.0 \\
\hline Omission of a drug from the physician's order (indication not treated) & Significant & 3 & 7.5 \\
\hline Omission of a preventive measure to avoid adverse reactions & Significant & 1 & 2.5 \\
\hline Omission of testing in drugs with possible hypersensitivity & Serious & 0 & 0.0 \\
\hline Adverse reaction with a threat to the life of the patient & Potentially lethal & 1 & 2.5 \\
\hline Adverse reaction related to precautions or contraindications & Serious & 2 & 5.0 \\
\hline Sequential therapy & Minor & 0 & 0.0 \\
\hline Route of administration that could lead to mild toxicity & Minor & 1 & 2.5 \\
\hline Route of administration that could lead to severe toxicity & Serious & 2 & 5.0 \\
\hline Information on the administration of parenteral/oral drugs & Significant & 2 & 5.0 \\
\hline
\end{tabular}

"Interventions related to errors involving drug overdose.

phase, the drugs for which consensus with the nursing staff was not reached in the first phase were evaluated based on drug consumption during the year 2009 in order to determine the need for availability in the unit. In these cases, data on drug consumption in 2009 were obtained from Farhos Gestión (a database used in the pharmacy department) for those medicinal products included in the Pyxis ADS on the unit and that contained the same active substance; the products with the highest level of consumption during the previous year were selected and those that could lead to confusion were withdrawn, thus also reducing the likelihood of expiry of the medication. The profiles of some drugs were modified based on this information and the inventory of the ADS in the unit was updated. As a variable of the principal objective, the number of drugs whose profiles were modified was recorded. The relationship with the nursing team has facilitated the detection of problems related to the request, receipt and storage of drugs not held in the automatic store; these activities were defined as secondary objectives of the resident's rotation.

\section{Teaching activity}

Training needs were detected in both theoretical aspects (difficulty comprehending the morning sessions of the care team or nursing activities related to drug administration) and practical aspects (prescription validation and difficulties related to the identification and categorisation of NOMs). These aspects were assessed by a process of self-evaluation, periodic meetings with the head of the PICU and head of teaching, and comparison with the schedules from countries that provide specific training programmes in this discipline and the activities normally carried out by pharmacists in our hospital.

\section{Results}

\section{Clinical activities}

Forty pharmaceutical interventions were performed, representing about four interventions per 100 patient-days.

\section{NOM detected}

The profile of the interventions was as follows (figure 1): recommendations

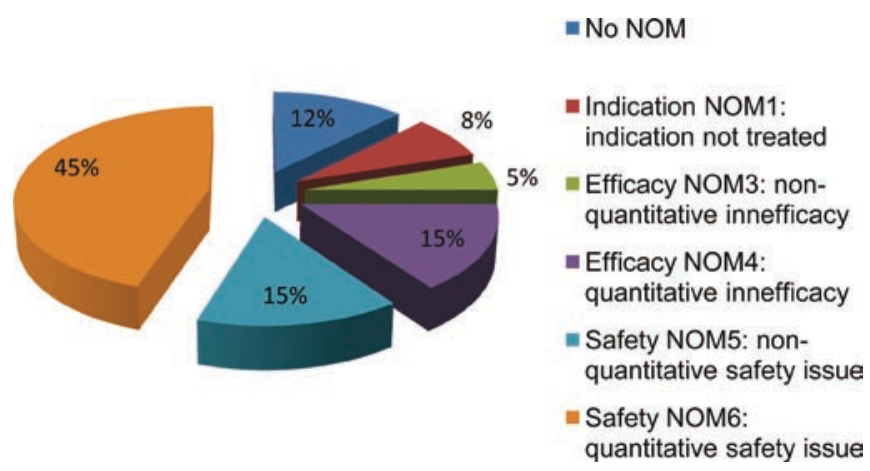

Figure 1 Stratification of the interventions by type of drug-related negative outcome. 
issued in the absence of error (12\%); problems regarding drug safety $(59 \%$; $44 \%$ of all interventions were related to quantitative issues); efficacy issues (20\%); and recommendations concerning the indication (8\%).

\section{Reason for the intervention and clinical significance}

The motives for the intervention (table 1) were related to errors due to overdosage in $17.5 \%$ of cases. The clinical significance of the pharmacist's recommendations is shown in figure 2 , with $72.5 \%$ being classified as significant or very significant. The pharmacist was on the ground at the time of the prescription. Hence, 100\% of the interventions were performed before drug administration.

Ten consultations were made by the physicians and nurses of the PICU, most of which were related to drug

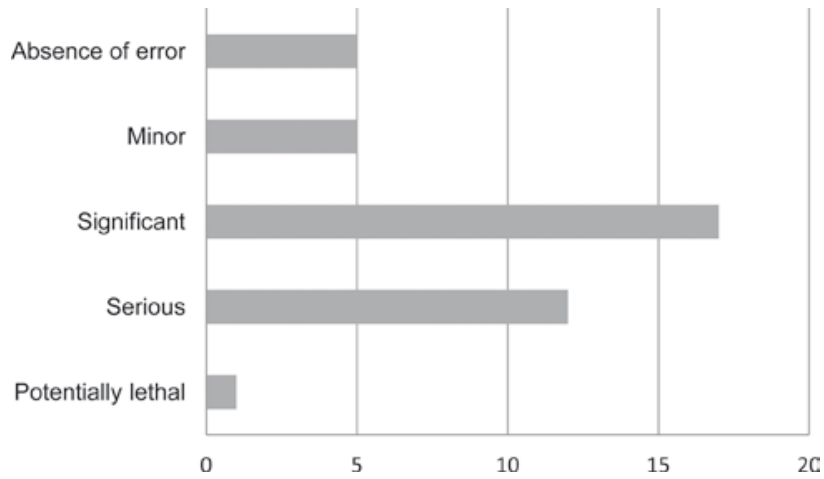

Figure 2 Number of interventions according to clinical significance.

administration. This coincided with the implementation of a drug administration guideline which led to a small improvement in this concept. Concerning pharmacovigilance, two medication errors were reported using the voluntary notification process.
Acceptance of the intervention

The acceptance rate obtained was 95\%.

Drug involved in the intervention

No single drug was involved in more than $7.5 \%$ of interventions. Table 2 shows interventions by active drug.

\begin{tabular}{|c|c|c|}
\hline Acetylcysteine & Clarification of the physician's order/request for information & No NOM \\
\hline Acetylcystein & Omission of a drug from the physician's order (indication not treated) & Untreated health problem \\
\hline Acetylcysteine & Clarification of the physician's order/request for information & Non-quantitative ineffectiveness \\
\hline Acyclovir & Dose error 1.5-10 times higher than normal (if drug had a normal therapeutic range) & Quantitative safety problem \\
\hline Albumin & Pharmaceutical form inappropriate or not available & Quantitative ineffectiveness \\
\hline Azithromycin & Clarification of the physician's order/request for information & Quantitative safety problem \\
\hline Calcium (oral) & Pharmaceutical form inappropriate or not available & No NOM \\
\hline Clonidine & Inappropriate dosage interval & Quantitative safety problem \\
\hline Clopidogrel & Clarification of the physician's order/request for information & No NOM \\
\hline Dexamethasone & Incomplete information in the physician's order & Quantitative safety problem \\
\hline Dopamine & Dose error 10 times higher than normal (if drug had a narrow therapeutic range) & Quantitative safety problem \\
\hline Esomeprazol & Inappropriate dosage interval & Quantitative safety problem \\
\hline Ethomidate & Dose error 4-10 times higher than normal (if drug had a narrow therapeutic range) & Quantitative safety problem \\
\hline Furosemide & Inappropriate dosage interval & Quantitative safety problem \\
\hline Hydrocortisone & Clarification of the physician's order/request for information & Quantitative safety problem \\
\hline Latex & Documented drug allergy & No NOM \\
\hline Levosimendan & Clarification of the physician's order/request for information & Non-quantitative ineffectiveness \\
\hline Magnesium & Dose error resulting in potentially toxic concentrations & Quantitative safety problem \\
\hline Nifedipine & Therapeutic duplication & Unnecessary medicine \\
\hline Nitazoxanide & Omission of a drug from the physician's order (indication not treated) & Non-quantitative ineffectiveness \\
\hline Octreotide & Information on the administration of parenteral/oral drugs & Non-quantitative ineffectiveness \\
\hline Octreotide & Information on the administration of parenteral/oral drugs & Non-quantitative safety problem \\
\hline Omeprazole & Inappropriate dosage interval & Quantitative safety problem \\
\hline Omeprazole & Inappropriate dosage interval & Quantitative safety problem \\
\hline Omeprazole & Inappropriate dosage interval & Quantitative safety problem \\
\hline Omeprazole & Interaction: clinically significant, requiring follow-up & No NOM \\
\hline Oseltamivir & Dose error very low in non-potentially life-saving drugs & Quantitative ineffectiveness \\
\hline Oseltamivir & Dose error very low in non-potentially life-saving drugs & Quantitative ineffectiveness \\
\hline Palivizumab & Omission of a drug from the physician's order (indication not treated & Untreated health problem \\
\hline Propranolol & Dose error 10 times higher than normal (if drug had a normal therapeutic range) & Quantitative safety problem \\
\hline Propofol & Adverse reaction related to precautions or contraindications & Quantitative safety problem \\
\hline Propofol & Adverse reaction related to precautions or contraindications & Quantitative safety problem \\
\hline Propofol & Adverse reaction with a threat to the life of the patient & Quantitative safety problem \\
\hline Quinine & Omission of a preventive measure to avoid adverse reactions & Non-quantitative safety problem \\
\hline Salbutamol & Route of administration that could lead to severe toxicity & Non-quantitative safety problem \\
\hline Salbutamol & Route of administration that could lead to severe toxicity & Quantitative safety problem \\
\hline Socium chloride & Route of administration that could lead to mild toxicity & Non-quantitative safety problem \\
\hline Sodium bicarbonate & Dose error resulting in potentially toxic concentrations & Quantitative safety problem \\
\hline Suxamethonium & Dose error resulting in potentially toxic concentrations & Quantitative safety problem \\
\hline Valproic acid & Interaction clinically significant, requiring follow-up & Quantitative ineffectiveness \\
\hline
\end{tabular}

NOM, negative outcomes associated with medication. 


\section{Logistic activities}

Results of the primary objective Forty-eight of the 289 medicinal products held in the ADS underwent changes to their prescription profile or were removed from the dispensary cabinet. These modifications were based on stock/ consumption management criteria or safety issues to avoid possible confusion with other drugs commonly used in the unit (table 3).

\section{Results of the secondary objectives} Improvements were made at two levels. The first involved improvements in the drug circuits (there had been many reports of incidents in the receipt of requests for enteral nutrition) and review of the expiry dates of drugs not held in the ADS of the unit. The second involved information management: recommendations on the choice of various pharmacyprepared formulations, resolution of doubts concerning the use of electronic prescriptions, detection of deficiencies in the prescription system and potentiation of its use in accordance with hospital policy.

\section{Areas for improvement in teaching}

After the pharmacist's rotation in the unit it was considered necessary to present proposals to improve the clinical rotation (table 4).

\section{Discussion}

Forty pharmaceutical interventions were performed, representing about 4.0 interventions per 100 patient-days. The number of interventions observed in similar studies varies and comparisons are often difficult owing to the numerous factors that affect the methodology of studies (such as the number and type of patients seen, number and level of specialisations of the pharmacists involved and system for recording interventions) and also to the fact that the results are presented using different types of activity indicators. Studies at a national level in the adult population have reported numbers of interventions that vary between 0.70 and 3.2 per 100 patientdays. ${ }^{12} 13$ The study by Izco et a ${ }^{14}$ specified a dedication of $5 \mathrm{~h}$ per day to the review of admitted patients and recorded a total of 3.23 interventions per 100 patient-days. Some international studies have reported higher levels of intervention in an adult population, with ranges from 1.2 to 11.5 interventions per 100 patient-days. ${ }^{1516}$ No Spanish studies in a paediatric population have quantified the activity indicators using interventions per 100 patient-days. In the USA, Folli and colleagues described interventions in various paediatric areas in two hospitals, ${ }^{17}$ reporting figures of 1.35 1.77 interventions per 100 patient-days; in that study, the PICUs had the highest risk of medication errors due to the wide range of weights and heights of the patients admitted to the units and to the large number of drugs used.

This variability in reported intervention levels may also be due to the fact that in some studies the recorded interventions included drug consultations and the clarification of physicians' orders due to illegibility. In our study, consultations on drug administration (mostly intravenous) were recorded but they were quantified separately from the interventions.

\begin{tabular}{|c|c|c|}
\hline \multicolumn{3}{|l|}{ Drug profile } \\
\hline \multicolumn{2}{|c|}{ Drugs predefined as URGENT + PRN GROUP (group 2) } & $(n=88)$ \\
\hline & Number of drugs modified & 3 \\
\hline \multirow[t]{2}{*}{ Drugs withdrawn" } & $\begin{array}{l}\text { Percentage of drugs modified in this } \\
\text { group }\end{array}$ & $3.4 \%$ \\
\hline & Number of drugs modified & 15 \\
\hline Drugs categorised as ' $\mathrm{e}^{\prime \dagger}$ & $\begin{array}{l}\text { Percentage of drugs modified in this } \\
\text { group }\end{array}$ & $17.05 \%$ \\
\hline \multicolumn{2}{|c|}{ Drugs predefined with profile of PRESCRIPTION ONLY (group 1) } & $(n=201)$ \\
\hline & Number of drugs modified & 4 \\
\hline Drugs withdrawn & $\begin{array}{l}\text { Percentage of drugs modified in this } \\
\text { group }\end{array}$ & $1.99 \%$ \\
\hline \multirow[t]{2}{*}{ Drugs categorised as ' $\mathrm{e}^{\prime}$ (a) } & Number of drugs modified & 24 \\
\hline & $\begin{array}{l}\text { Percentage of drugs modified in this } \\
\text { group }\end{array}$ & $11.94 \%$ \\
\hline \multicolumn{2}{|c|}{$\begin{array}{l}\text { Drugs categorised as 'e' to avoid medication errors despite not being high } \\
\text { consumption or very urgent medicinal products }\end{array}$} & $(n=0)$ \\
\hline \multicolumn{2}{|l|}{ Number of drugs } & 2 \\
\hline \multicolumn{2}{|c|}{ Percentage of drugs modified in this group } & $0.99 \%$ \\
\hline
\end{tabular}

Furthermore, the use of the electronic prescription in $100 \%$ of cases avoided illegibility of the physicians' orders, and the category 'clarification of physician's orders' was therefore used to refer only to those cases in which information was requested because the validator considered that the nursing staff could misinterpret the prescription, giving rise to an error of drug administration. In addition, the overlap of this study with the initiation of the pharmacokinetics service in our Pharmacy Department made it impossible to include the consultations and reports issued by this service as interventions; in some studies, these interventions have represented a high percentage of all the interventions recorded. ${ }^{16}$

With regard to the distribution of drugrelated problems, the results are similar to those of the study performed in Argentina by Fontana et a ${ }^{18}$ in which $52.5 \%$ of the problems were related to drug safety, $32.5 \%$ to efficacy and $15 \%$ to indication. As in our study, those authors found the highest number of interventions to be related to safety issues. The majority of interventions are therefore related to the detection of errors of prescription that constitute an absolute overdose of drugs, a common event in paediatric units and intensive care units. Although requests for information are the most common source of interventions in some studies, ${ }^{19}$ overdosage continues to be one of the most frequent reasons for pharmacist intervention. ${ }^{4}$ 2021 Our data support those of other authors who report a significant number of prescriptions with doses two, three or up to 10 times the dose recommended in children. ${ }^{22} 23$

Although there was no single active substance that accounted for the majority of these interventions, it must be realised that our database did not group the drugs involved into the therapeutic groups to which they belong. The study by Folli et a $11^{17}$ found that the majority of potentially serious or fatal errors occurred with antibiotics, theophyllines, digoxin and fluid therapy. Furthermore, studies undertaken in adult intensive care units highlight certain therapeutic groups as the source of the majority of interventions. ${ }^{24}$ Analysis of these data would be useful in order to identify prescription patterns and common errors that require correction through pharmacist intervention.

The rate of acceptance by physicians in our study (95\%) was similar to that of other studies which have reported acceptance rates of $91-99 \%$, and no inappropriate interventions were detected. ${ }^{1425-27}$ 
Table 4 Areas for improvement in teaching

Phase 1: Prior to the rotation: theoretical capacity

Acid-base balance

Analgesiasedation

Enteral and parenteral nutritionPulmonary hypertension

Phase 2: During the rotation

Review the basic physiopathological disturbances in patients admitted to the unit and the pharmacological treatment of those disturbances

Establish routine attendance at the sessions. Obligatory attend ance at the 09:00 morning session and encourage attendance at the 14:15 session

Possibility for establishing a session given by the pharmacist on a subject of interest to the unit

Substitution of the general pharmacy literature reviews through a review of articles on the subject to be presented

Review of the use of antimicrobials in critically ill paediatric patients

Selection of the course sessions given in the ICU. To gain greatest benefit from the course, it should be performed 1 month after beginning the rotation

Note: It would be interesting for the resident to participate in the session on electronic prescription
Key issues in shift changes. Very useful to facilitate understanding of the decisions that are taken according to the patient's biochemical and clinical parameters

With regard to pharmacotherapy, they enable the resident to focus on the important aspects and differentiate the problems associated with the medication that could arise in an intensive care unit from those that may occur in other types of hospital ward

Detect the key parameters for performing pharmacotherapeutic follow-up of patients. Know the basic parameters for detecting the response to pharmacological treatment

Clarify on the same day any doubts that arise regarding validation of the orders. Understanding of the clinical course of the patients

Training of the resident in a specific subject and integration of the pharmacist as a member of the care team

Acquisition of an understanding of antibiotic therapy and observation in clinical practice

Obligatory

- Fluid therapy

- Acid-base balance

- Parenteral and enteral nutrition

- Inotropic and vasodilator drugs, volume expanders, and diuretics

- Analgesia, sedation, and muscle relaxants

- Acute renal failure: extrarenal filtration techniques

- Infection: empirical antibiotic therapy

Very interesting.

- Ventilators and mechanical ventilation (attend at least one of the sessions)

- Haematological disturbances

- Coma, status epilepticus, cerebral protection

Detection of strong and weak points of the rotation

Self-evaluation of proposed objectives and of those achieved

Report of the activities performed

With respect to the results of the logistic activities, it was difficult to compare findings as no publications have quantified the duties undertaken by that the pharmacist to optimise the ADS, although evaluations of the impact of the introduction of automatic cabinets and of the electronic prescription system on the reduction in medication errors have been performed. ${ }^{28} 29$

The principal limitation of our study is that the data correspond to the activity of a single resident pharmacist whose dedication was not completely uniform over the 3 months of the rotation and whose training in general paediatrics was performed in parallel with her training in paediatric intensive care. Although the English-speaking countries have developed specific training schedules, ${ }^{30} 31$ it is necessary for each country to design their own schedules according to pharmacist availability and level of integration into the care team, with adaptation to each hospital. Our contribution to the improvement in training could give rise to the creation of an initial programme in those hospitals that have not yet implemented this activity in a highly specialised unit such as a PICU. ${ }^{32} 33$ Our level of understanding of paediatrics and the complexity of these patients makes it difficult to identify a large number of true NOMs, leading to the need for more detailed pharmacotherapeutic patient monitoring.

\section{Conclusions}

The integration of a fourth-year resident into the multidisciplinary team in the PICU facilitates the detection of drug-related problems and enables the pharmacotherapy of critically ill paediatric patients to be optimised. Furthermore, the close relationship with the nursing staff and acquisition of additional information about the problems faced by health professionals in the unit each day has facilitated resolution of events and the identification of areas for improvement in the processes related to the request, receipt and optimisation of use of drugs. However, the integration of the pharmacist into the care team depends directly on the usefulness that the pharmacist demonstrates through these activities, making it essential to broaden the study to include pharmacoeconomic issues (differences in the costs of treatment after the pharmacist's intervention) to quantify the economic saving, which could lead to an increase in the number of pharmacists who could transfer their activities to the clinical setting.

Acknowledgements The authors are very grateful to all the staff of the intensive care unit for their enthusiastic cooperation. They thank all the staff physicians and residents of the unit for the explanations received and for their teaching and give particular thanks to the nursing staff for their unreserved collaboration.

Contributors LE-M was the lead author and wrote the final version of the manuscript. She designed the study, working with the other authors to define the variables, and also carried out the overall statistical analysis of the data. CMF-L, SM-R and IG-L helped to design the study, defined the variables and checked the statistical 
analyses, and critically reviewed the text. JL-H and MS-S reviewed the final text.

Ethics approval Ethics approval was obtained from the paediatric intensive care unit.

Provenance and peer review Not commissioned; externally peer reviewed.

\section{References}

1. Giráldez J, Aldaz A. Docencia en Farmacia Hospitalaria. In: Farmacia Hospitalaria, eds. Fundación Española de Farmacia Hospitalaria. 3rd edn Madrid: Doyma, 2002:363-88.

2. Castro Cels I. Continuous training in pharmacotherapy for specialists in hospital pharmacy. Farm Hosp 2007;31:48-66.

3. Calleja Hernández MA. Líneas estratégicas de formación en la Sociedad Española de Farmacia Hospitalaria (SEFH). Farm Hosp 2008;32:305-8.

4. Kaushal R, Bates DW, Abramson EL, et al. Unitbased clinical pharmacists' prevention of serious medication errors in pediatric inpatients. Am J Health Syst Pharm 2008;65:1254-60.

5. Preventing pediatric medication errors [homepage on the Internet] 2008. http://www. jointcommission.org/assets/1/18/SEA_39.pdf (accessed 17 Jan 2010).

6. Krupicka MI, Bratton SL, Sonnenthal K, et al. Impact of a pediatric clinical pharmacist in the pediatric intensive care unit. Crit Care Med 2002;30:919-21.

7. Franke HA, Woods DM, Holl JL. High-alert medications in the pediatric intensive care unit. Pediatr Crit Care Med 2009;10:85-90.

8. American Society of Health-System Pharmacists. ASHP guidelines on the safe use of automated medication storage and distribution devices. AmJ Health Syst Pharm 1998;55:1403-7.

9. Fernandez-Llamazares CM, Manrique-Rodriguez S, Perez-Sanz C, et al. Validation of a method for recording pharmaceutical interventions. J Clin Pharm Ther Published Online First: 28 Dec 2011. doi: 10.1111/j.1365-2710.2011.01328.x.
10. Panel de consenso. Tercer consenso de Granada sobre Problemas Relacionados con Medicamentos (PRM) y Resultados Negativos asociados a la Medicación (RNM). Ars Pharm 2007:48:5-17.

11. Overhage JM, Lukes A. Practical, reliable, comprehensive method for characterizing pharmacists' clinical activities. Am J Health Syst Pharm 1999:56:2444-50.

12. Cuenca ML, Aguilar T, Desongles T, et al. Cambios en la terapéutica tras la intervención del farmacéutico. Farm Clin 1998;15:76-82.

13. Farré Riba R, Clopés Estela A, Sala Esteban $M L$, et al. Intervenciones farmacéuticas (parte I): metodología y evaluación. Farm Hosp 2000;24:136-44

14. Izco N, Codina C, Tuset M, et al. Evaluación de la integración del farmacéutico en equipos de atención de las unidades de hospitalización. Farm Hosp 2002:26:18-27.

15. Shane R, Saltiel E, White JY, et al. Using documentation of pharmacists' clinical activity. Am J Hosp Pharm 1991;48:2647-8.

16. Chin JM, Muller RJ, Lucarelli CD. A pharmacy intervention program: recognizing pharmacy's contribution to improving patient care. Hosp Pharm 1995;30:120, 123-6, 129-30.

17. Folli HL, Poole RL, Benitz WE, et al. Medication error prevention by clinical pharmacists in two children's hospitals. Pediatrics 1987;79:718-22.

18. Fontana Raspanti D, Solá Uthurry N. Seguimiento farmacoterapéutico en pacientes pediátricos hospitalizados: adaptación de la metodología Dáder. Farm Hosp 2003;27:78-83.

19. Ghaleb MA, Barber N, Franklin BD, et al The incidence and nature of prescribing and medication administration errors in paediatric inpatients. Arch Dis Child 2010;95:113-18.

20. Jonville AP, Autret E, Bavoux F, et al. Characteristics of medication errors in pediatrics. DICP 1991;25:1113-18.

21. Kozer E, Scolnik D, Macpherson A, et al. Variables associated with medication errors in pediatric emergency medicine. Pediatrics 2002;110:737-42.
22. Koren G, Haslam RH. Pediatric medication errors: predicting and preventing tenfold disasters. J Clin Pharmacol 1994;34:1043-5.

23. Koren G, Barzilay Z, Greenwald M. Tenfold errors in administration of drug doses: a neglected iatrogenic disease in pediatrics. Pediatrics 1986;77:848-9.

24. Arroyo Conde C, Aquerreta I, Ortega Eslava A, et al. Clinical and economic impact of the pharmacy resident incorporation into the healthcare team. Farm Hosp 2006;30:284-90.

25. Virani A, Crown N. The impact of a clinical pharmacist on patient and economic outcomes in a child and adolescent mental health unit. Can J Hosp Pharm 2003;56:158-62.

26. Gorgas Torner MQ, Odena Estrade E, Pastor Solernou F. Pharmaceutical care for drug-related problems in inpatients. Farm Hosp 2003;27:280-9.

27. Montazeri M, Cook DJ. Impact of a clinical pharmacist in a multidisciplinary intensive care unit. Crit Care Med 1994;22:1044-8.

28. King WJ, Paice N, Rangrej J, et al. The effect of computerized physician order entry on medication errors and adverse drug events in pediatric inpatients. Pediatrics 2003;112(Pt 1):506-9.

29. Wang JK, Herzog NS, Kaushal R, et al. Prevention of pediatric medication errors by hospital pharmacists and the potential benefit of computerized physician order entry. Pediatrics 2007;119:e77-85.

30. Martin KA. Specialized pediatric residency programs. Am J Health Syst Pharm 2005;62:1766-8.

31. Koren $\mathrm{G}$. Training clinicians in pediatric pharmacology-toxicology: the Toronto model. Paediatr Drugs 2009;11:60-2.

32. Echarri-Martinez L, Fernandez-Llamazares CM, Lopez-Herce J, et al. Paediatric training for developing clinical activities. Farm Hosp 2012;36:43-9.

33. Fernandez-Llamazares CM, HernandezGago Y, Pozas M, en representacion del Grupo Español de Farmacia Pediatrica. Paediatric pharmacy: training and information. Farm Hosp 2011;35:103-5. 